\title{
Plasma spheroidization of nickel powders in a plasma reactor
}

\author{
G SHANMUGAVELAYUTHAM and V SELVARAJAN* \\ Department of Physics, Bharathiar University, Coimbatore 641 046, India
}

MS received 19 July 2003; revised 19 June 2004

\begin{abstract}
Thermal spray coatings of surfaces with metal, alloy and ceramic materials for protection against corrosion, erosion and wear is an intense field of research. The technique involves injection of the powder into a plasma flame, melting, acceleration of the powder particles, impact and bonding with the substrate. Feedstock powders of metals, alloys and ceramics for thermal spray applications have to meet several requirements. Particle shape, size and its distribution, powder flow characteristics and density are the important factors to be considered in order to ensure high spray efficiency and better coating properties. For smooth and uniform feeding of powders into plasma jet, the powder particles have to be spherical in shape. High temperatures and steep temperatures present in thermal plasma is exploited to spheroidize particles in the present investigation. Nickel powder particles in the size range from $40-100 \mu \mathrm{m}$ were spheroidized using plasma processing. SEM and optical micrographs showed spherical shape of processed particles.
\end{abstract}

Keywords. Thermal plasma; spheroidization; nickel.

\section{Introduction}

Spherical nickel powders are widely used in a variety of domains like powder metallurgy, electronic industry, chemical processing, etc. In some of these applications, shape and size of the particles are among critical parameters and must be well chosen.

Fine powders are desired in forming and fabricating processes of high precision and complex shaped parts made of metals and ceramics. For example, fine spherical powders with diameter of $<20 \mu \mathrm{m}$ are essential to the success of powder injection moulding, where powder flowability is important (Lawley 1989). Smaller diameter particles are sintered easily to high density, while the microstructure and chemical homogeneity may be enhanced also. The quest for novel microstructures with improved high temperature properties has accentuated the development of rapidly solidified materials. These materials provide unique microstructures, enhanced homogeneity, and thorough new phase reaction sequences. The use of rapidly solidified powders in conjunction with traditional powder metallurgy processing has resulted in components having enhanced high temperature performance and the additional capability of generating neat - netshape components, which helps to conserve scarce raw materials.

There are several methods of producing rapidly solidified powders with quench rates in the range $10^{2}-10^{6} \mathrm{~K} \mathrm{~s}^{-1}$, these including gas and water atomization (Small and Bruce 1968; Lubanska 1970), melt spinning (Libermann 1980),

*Author for correspondence laser melting and quenching (Breinan and Kear 1978), and plasma deposition (Gerdeman and Hecht 1972). Gas atomization, using either inert gas or air, is one of the commonly used atomization methods for producing spherical powders (Dunkley 1990). Other atomization processes, which have been used in the production of rapidly solidified powders include ultrasonic atomization, centrifugal atomization, rotating electrode atomization and roller atomization (Lawley 1977). Plasma processing is the most versatile technique for spheroidization of metal and ceramic powder particles. In this paper production of spherical nickel powders using the d.c. plasma reactor is reported.

Such products, characterized by an irregular shape and a strong tendency to agglomerate to chain like form, can be classified by Geldart classification (Geldart 1973) as group c particles meaning difficulty of feeding. Plasma densification of such agglomerated particles produce spherical particles with a size many times their original individual diameter. For this reason, the feeding method and its operating conditions are important parameters that have to be properly controlled in order to obtain the desired product.

The plasma environment offers a number of other possibilities for materials processing (Tetronics 2002):

- Reactive processing to produce nitride and other ceramic particles

- Spheroidization of facetted particles

- Particle size reduction by evaporation-condensation

- Surface coating by deposition or surface reaction

- Heat treatment and phase changes

- Use of the plasma environment to synthesis metastable phases or extend solid solubility. 
The methods of plasma processing, in particular those aimed for thermal treatment of powder (spheroidization, densification and purification) use either arc or inductive plasma torches (Pfender 1997). High temperatures $\left(10^{4} \mathrm{~K}\right)$ and steep temperature gradients $\left(10^{6} \mathrm{~K} / \mathrm{m}\right)$ present in the thermal plasmas can be exploited to produce spherical particles [Spherical particles are formed by partially melting the surface of the particles in the plasma flame and rapid quenching]. In the present investigation a d.c. non-transferred plasma torch is used to generate a thermal plasma jet.

\section{Experimental}

\subsection{Plasma reactor}

The experimental set-up consists of a $40 \mathrm{~kW}$ d.c. plasma spray torch (Ion Arc machines (I) Pvt. Ltd) mounted on a water-cooled reaction chamber made of stainless steel.

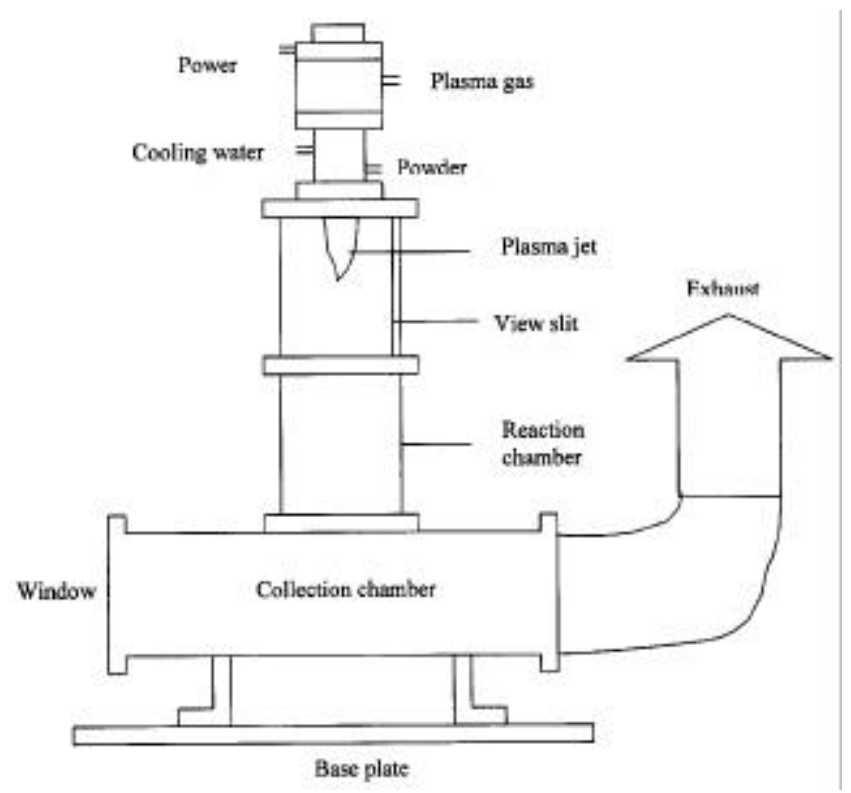

Figure 1. Schematic diagram of a plasma reactor.
The reaction chamber is 6" in diameter and $24 "$ long. The powder is collected in a water-cooled crucible kept at the bottom of the reaction chamber (figure 1). A schematic of the experimental set-up is shown in figure 2. The set-up also includes a d.c. power supply (75 V, 600 A), H.F. igniter, gas feed system, water cooling system and a powder feeder. A control console controls gas and water flow rates.

The plasma torch was ignited by striking a high current arc between the cathode and anode (nozzle) and the desired power level was maintained by controlling the arc current and the flow rate of plasma gas. A mixture of argon and nitrogen was used as the plasma forming gas and argon gas used as the carrier gas. Nickel powder was fed into the plasma by means of a screw type gravimetric powder feeder. The powder particles fed into the plasma flame get accelerated and melt simultaneously. The particles leave the flame and get quenched. During the process the molten particles get spheroidized due to surface tension. Typical operating parameters are given in table 1. The processed powder was characterized by X-ray diffraction for phase composition and optical microscope for particle morphology.

Microstructural parameters such as grain size $(D)$, microstrain $(\varepsilon)$ and dislocation density $(\delta)$ are calculated from Scherrer's formula from the FWHM of the peaks expressed in radians (Cullity 1972; Suryanarayana 2001),

$$
\begin{aligned}
& D=\frac{0 \cdot 94 \lambda}{\beta \cos \theta}, \\
& \delta=1 / D^{2},
\end{aligned}
$$

and the relation (Willamson and Smallman 1956)

$$
\varepsilon=\left(\frac{\lambda}{D \cos \theta}-\beta\right) x \frac{1}{\tan \theta},
$$

where $\lambda$ is the wavelength of the X-ray and $\beta_{2 \theta}$ is the full width at half maximum of the corresponding peak.

The products manufactured using this technique is well adapted to thermal spraying because of the following pro-

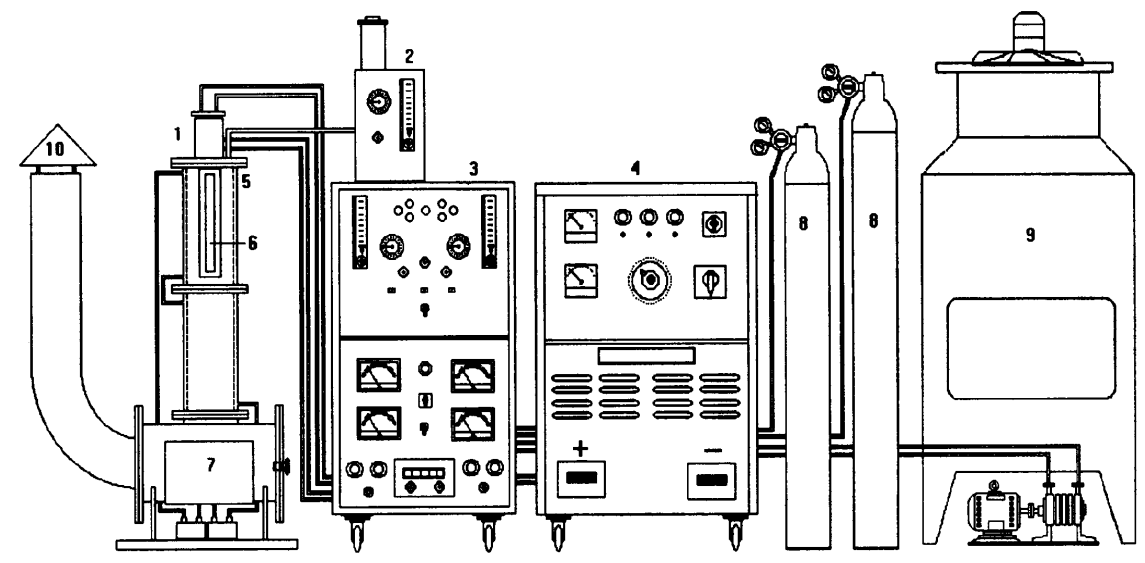

Figure 2. Schematic diagram of plasma processing set-up (1. Plasma torch; 2. powder feeder; 3. control console; 4. plasma power source; 5. plasma reactor; 6. view window; 7. powder collection chamber; 8 . gas cylinders; 9 . cooling tower and 10. exhaust). 
perties: (i) The size and granulometric distribution is easily controllable, (ii) good flowability, (iii) suitable morphology, and (iv) homogeneous chemical composition.

\section{Results and discussion}

Results of the investigations are illustrated in figures 3-8 and table 2. The structural characteristics were studied through XRD measurements. XRD pattern for nickel powder before processing is exhibited in figure 3 . Figure 4 shows the comparison of the diffraction patterns processed at different input power levels. In figure 3, intensity peaks observed are due to nickel only. The structure of the nickel powder is found to be cubic in nature and it oriented along (111) direction. In figure 4, after processing, in addition to nickel peaks, intensity peaks due to nickel oxide are seen indicating that nickel oxide has been formed in the process of spheroidization. The structure of the nickel oxide is found to be a combination of cubic and hexagonal and its orientation along (200), (220),

Table 1. Operating parameters for d.c. plasma spheroidization $(\mathrm{Ni})$.

\begin{tabular}{ll}
\hline Torch & $: 40 \mathrm{~kW}$ d.c. plasma torch \\
Power input & $: 8,10,12$ and $14 \mathrm{~kW}$ \\
Plasma gas (argon) & $: 20 \mathrm{lpm}$ \\
Plasma gas (argon-nitrogen) & $: 20-3 \mathrm{lpm}$ \\
Anode cooling water flow rate & $: 12 \mathrm{lpm}$ \\
Cathode cooling water flow rate & $: 10 \mathrm{lpm}$ \\
& \\
Powder feeder: Gravimetric feeder & \\
Carrier gas (argon) & $: 3 \mathrm{lpm}$ \\
Powder feed rate & $: 9(\mathrm{~g} / \mathrm{min})$ \\
Quenching medium & $:$ Air \\
\hline
\end{tabular}

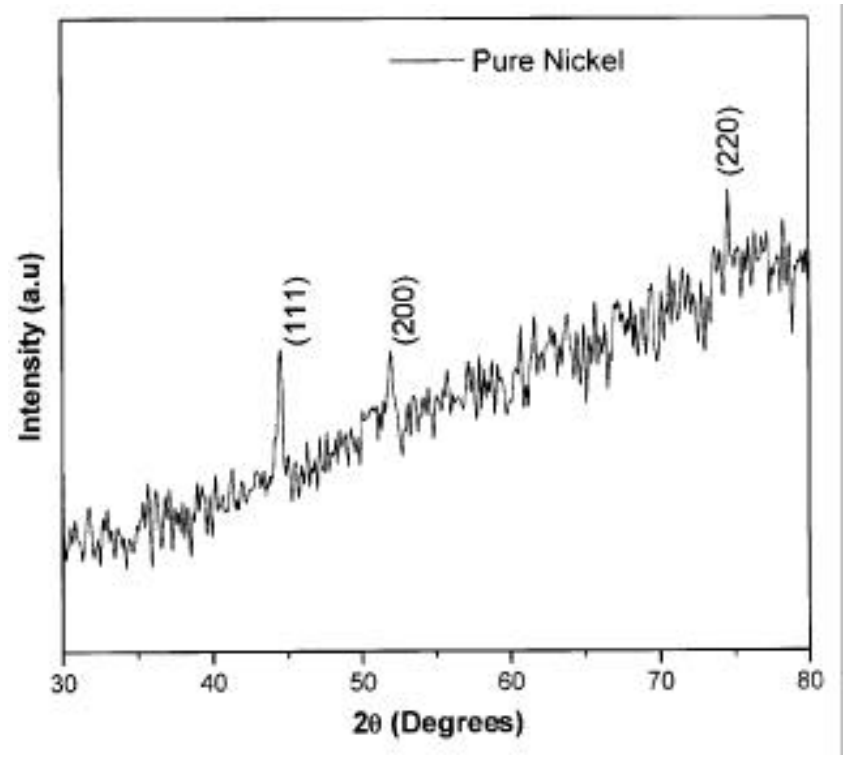

Figure 3. XRD pattern of pure nickel powder before plasma processing. and (112) direction. However, the intensity is found to increase with the increase in input power levels. Depending on the level of formation of $\mathrm{NiO}$ and orientation of the planes, the intensity of peaks is different at different power levels.

Grain size, dislocation density and strain of the processed powders are calculated from figure 4 . The calculated values are shown in table 2 . From this we concluded that the grain size values mainly depend on the input power levels. It is seen that increase in grain size with increase in input power levels, decreases the dislocation density and strain.

SEM photograph of the nickel powder before processing is shown in figure 5. It is clearly seen that particles are in different shapes (irregular, rounded). The particle size ranges from $40-100 \mu \mathrm{m}$. The optical micrograph of

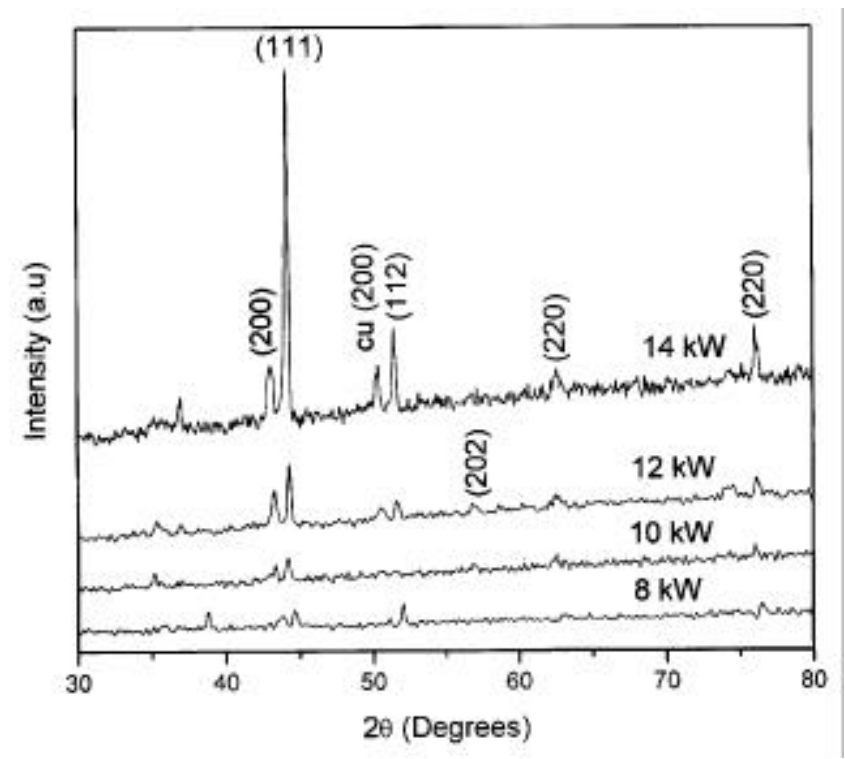

Figure 4. XRD patterns of processed nickel powders at different power levels.

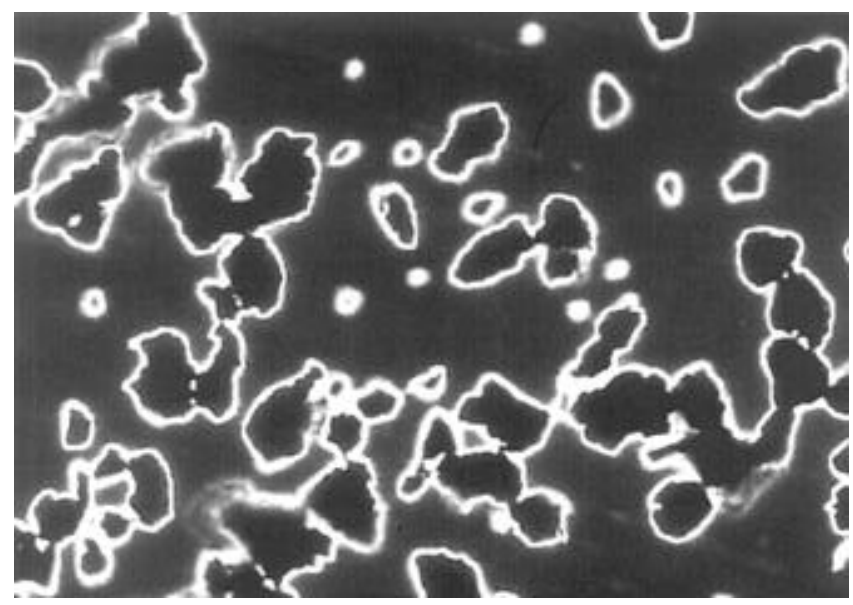

Figure 5. Optical photograph of nickel powder (before processing). 
nickel powder particles processed at different power levels are shown in figures 6,7 and 8 . At $8 \mathrm{~kW}$ power level there is no significant formation of spherical particles. At $10 \mathrm{~kW}$ power level some particles are spheroidized and agglomeration of particles is also seen. At $12 \mathrm{~kW}$ power level more number of spherical particles are seen and at $14 \mathrm{~kW}$ power level all the particles are seen to be spheroidized. When the particle is injected into the plasma flame, the surface of the particle is initially heated and get melted. Melting of the surface of the particles percolate deeper into the particle when the temperature increases. Hence when melting takes place deeper into
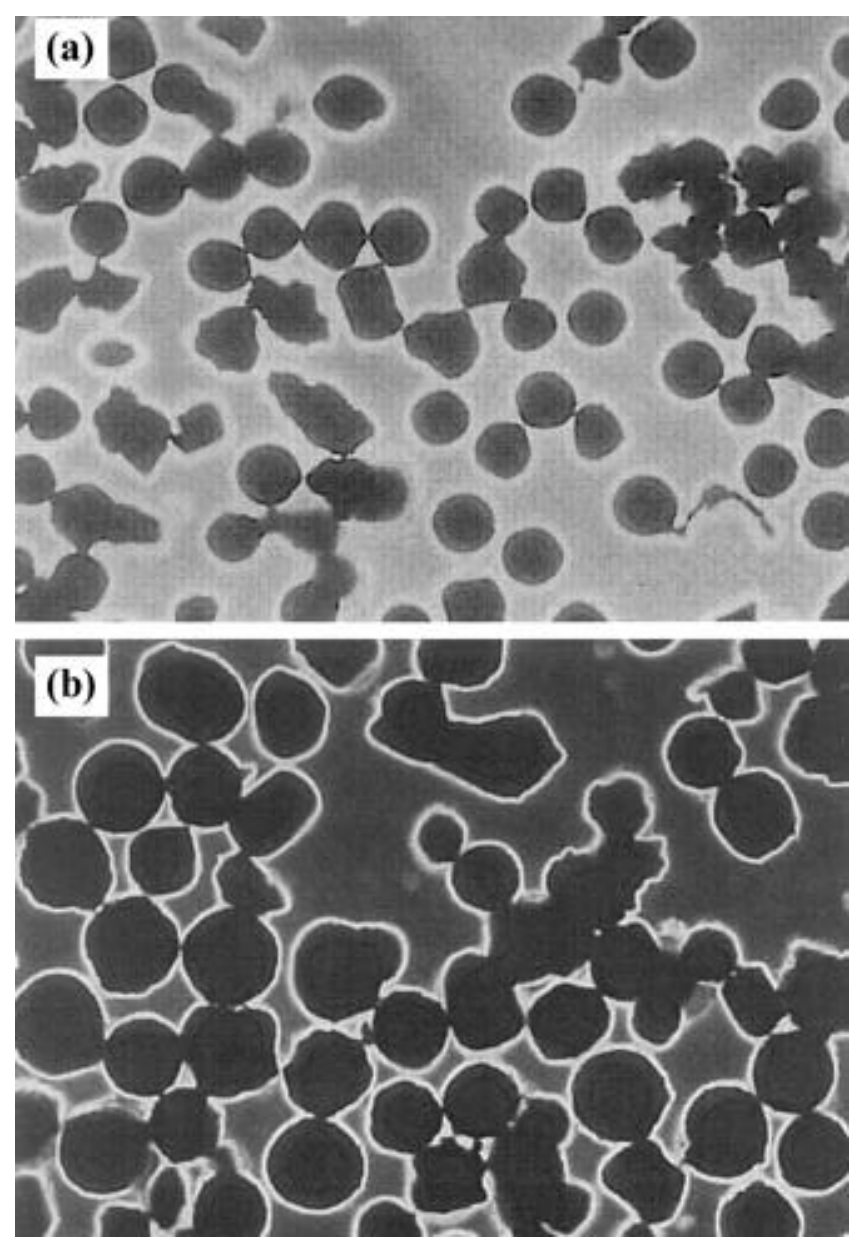

Figure 6. Optical photograph of nickel powders at two different power levels $(100 \times)$ : (a) Ni1-8 $\mathrm{kW}$ and (b) Ni2-10 kW. the particle, spheroidization is better. In the present investigation spheroidization of nickel powder is best at $14 \mathrm{~kW}$ power level.

\section{Conclusions}

Nickel powder particles in the size range from $40-100 \mu \mathrm{m}$ have been spheroidized using plasma processing. At a

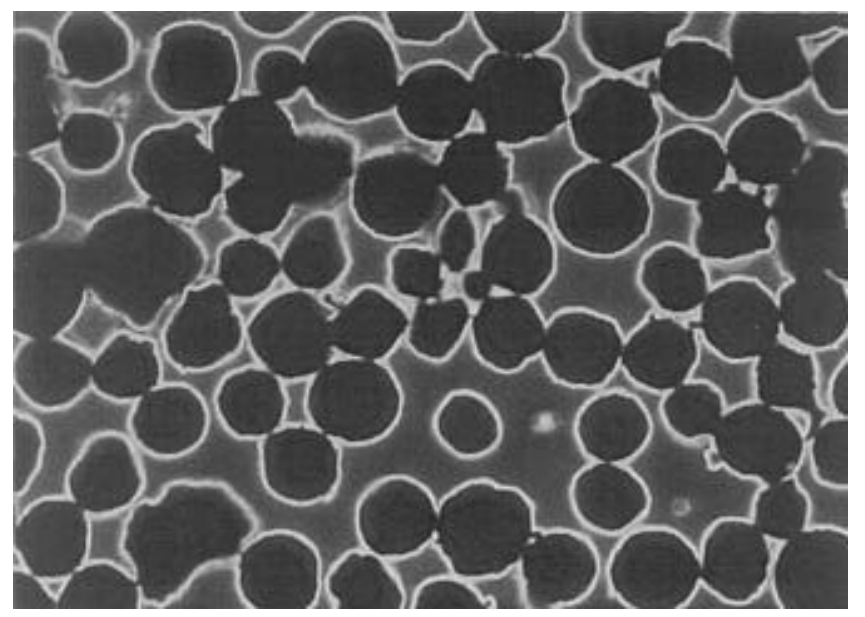

Figure 7. Optical photograph of nickel powders at $12 \mathrm{~kW}$ power level $(\mathrm{Ni5}-100 \times)$.

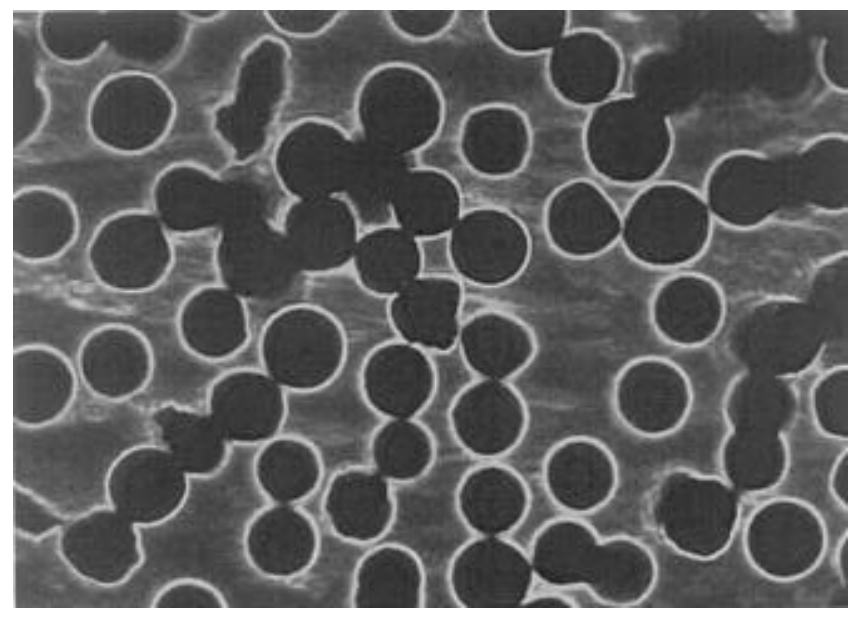

Figure 8. Optical photograph of nickel powders at $14 \mathrm{~kW}$ power level $(\mathrm{Ni7}-100 \times)$.

Table 2. Grain size, dislocation density and strain of processed nickel powder.

\begin{tabular}{lccccc}
\hline $\begin{array}{l}\text { Power } \\
(\mathrm{kW})\end{array}$ & $\begin{array}{c}2 \theta \\
\text { (Degrees) }\end{array}$ & $h k l$ & $\begin{array}{c}\text { Grain } \\
\text { size }(\mathrm{nm})\end{array}$ & $\begin{array}{c}\text { Dislocation } \\
\text { density }\left(\times 10^{15}\right) \\
\left(\operatorname{lin} / \mathrm{m}^{2}\right)\end{array}$ & $\begin{array}{c}\text { Strain }\left(\times 10^{-3}\right) \\
\left(\operatorname{lin}^{-2} \mathrm{~m}^{-4}\right)\end{array}$ \\
\hline 8 & 44.66 & $(111)$ & 21.29 & $2 \cdot 21$ & 2.48 \\
10 & $44 \cdot 19$ & $(111)$ & $25 \cdot 60$ & 1.53 & 0.95 \\
12 & $44 \cdot 18$ & $(111)$ & 30.90 & 1.04 & 0.80 \\
14 & 44.07 & $(111)$ & 34.45 & 0.84 & 0.71 \\
\hline
\end{tabular}


suitable input power level most of the particles were spheroidized. It was also observed that nickel oxide was formed in the process as exhibited from XRD analysis. It can be concluded that plasma processing is a successful method for spheroidization of micron sized particles. This study also shows that agglomeration of nickel particles can be minimized or eliminated by both powder feeding and plasma jet properties. Plasma temperature is an important parameter in obtaining good quality of spheroidization.

\section{Acknowledgements}

The authors gratefully acknowledge BRNS for funding the project. The authors wish to thank Dr N Venkatramani, Director, Beam Technology Development Group, Bhabha Atomic Research Centre, Mumbai, for his valuable suggestions, critical comments and to the Department of Metallurgy and Materials, P.S.G. College of Technology, Coimbatore, for extending SEM and optical microscope facilities.

\section{References}

Breinan E M and Kear B H 1978 in Rapid solidification processing principles and technologies (eds) R Mechrabian, B H Kear and M Cohen (Baton Rouge, LA: Claitors Publ.) p. 87

Cullity B D 1972 Elements of X-ray diffraction (MA: AddisonWesley Publishing Company Inc.) p. 102

Dunkley 1990 J. Met. Mater. 66361

Geldart D 1973 Powder Technology 7285

Gerdeman D A and Hecht N L 1972 Arc plasma technology in materials science (New York: Springer-Verlag)

Lawley A 1977 Int. J. Powder Metall. Powder Technol. 13 169

Lawley A 1989 ASM News 6

Libermann H H 1980 Technical information series, Report 80CRD117, General Electric Corp., Schenectady, NY

Lubanska H 1970 J. Met. 2245

Pfender E 1997 ISPC - 13 (Beijing, China) p. 3

Small S and Bruce T J 1968 Int. J. Powder Metall. 47

Suryanarayana C 2001 Prog. Mater. Sci. 4641

Tetronics 2002 Powder Metall. 458

Willamson G B and Smallman R C 1956 Philos. Mag. 134 